Revue internationale P.M.E.

Économie et gestion de la petite et moyenne entreprise

\title{
Pour une approche des spécificités financières de la PME au travers du concept de territoire financier
}

\section{Bernard Belletante}

Volume 4, numéro 1, 1991

URI : https://id.erudit.org/iderudit/1008025ar

DOI : https://doi.org/10.7202/1008025ar

Aller au sommaire du numéro

Éditeur(s)

Presses de l’Université du Québec

ISSN

0776-5436 (imprimé)

1918-9699 (numérique)

Découvrir la revue

Citer cet article

Belletante, B. (1991). Pour une approche des spécificités financières de la PME au travers du concept de territoire financier. Revue internationale P.M.E., 4(1), 49-79. https://doi.org/10.7202/1008025ar
Résumé de l'article

Si les spécificités de la PME ont fait l'objet de nombreux travaux dans les domaines de la stratégie et du marketing, il n'en est pas de même, en Europe, pour l'aspect financier. L'objet de cet article est donc de proposer un cadre théorique global permettant de repérer et de mesurer les spécificités financières des petites et moyennes entreprises. 


\title{
Pour une approche des spécificités financières de la PME au travers du concept de territoire financier
}

\author{
Bernard BELLETANTE* \\ Groupe ESC Lyon
}

\begin{abstract}
RÉSUMÉ
Si les spécificités de la PME ont fait l'objet de nombreux travaux dans les domaines de la stratégie et du marketing, il n'en est pas de même, en Europe, pour l'aspect financier. L'objet de cet article est donc de proposer un cadre théorique global permettant de repérer et de mesurer les spécificités financières des petites et moyennes entreprises.
\end{abstract}

\begin{abstract}
While the specific strategic and marketing issues of the SME have been treated in a large number of articles and papers, this has not been true, in Europe, of the financial aspects. The aim of this article, therefore, is to propose an overall theoretical framework, which makes it possible to identify and comprehend the specific financial aspects of the SME.
\end{abstract}

\section{RESUMEN}

Si las especificidades de las PyMEs han sido objeto de numerosos trabajos en los ambitos de las estrategia y del marketing, no occure la misma cosa, en Europa, desde el punto de vista financiero. Por consiguiente, el objeto de este articulo es el de proponer un marco teorico global que permita descubrir y evaluar las especifidades de las pequenas y medianas empresas.

* Professeur de finance. Adresse : ESC Lyon, 23 avenue Guy de Collongues, 69230 Ecully, France. 


\section{Introduction*}

Après une décennie marquée par l'éclosion d'innovations financières tous azimuts, juillet 1990 prendra date dans l'histoire financière européenne comme la constitution d'un marché européen des capitaux, comme un exemple parfait d'intégration financière et monétaire. Nous abandonnerons la problématique globale d'un processus d'intégration (Roure et Tabatoni, 1988) pour ne retenir qu'une des caractéristiques mise en avant pour la libéralisation des mouvements de capitaux en Europe : la diminution probable du coût des ressources financières et des services financiers, résultat d'une plus grande mobilité potentielle des capitaux dans les pays européens (de Boissieu 1987). L'explication majeure réside dans les comportements des différents offreurs et demandeurs de capitaux, lesquels vont aboutir à une égalisation du taux de rendement marginal du capital, égalisation provoquant pour les différents pays un gain global par rapport à l'existence de marchés financiers fragmentés (Peteers, 1989). Rappelons simplement que les innovations financières sont considérées comme un accélérateur de ce phénomène.

À partir de ce type d'approche théorique, il est aisé de construire un discours politique sur l'intérêt de tout agent économique à l'intégration des marchés (l'intégration étant comprise comme la vision technique de l'unicité du marché) et la Finance n'est d'ailleurs pas le seul champ d'application de cette vision ( $c f$. l'Europe des Transports, l'Europe sociale...). Une des conséquences directes de la prédominance de cette analyse est l'abandon par l'État (notamment en France) d'une réflexion et de la définition de moyens d'actions propres à un statut de la PME. «Il y a dix ans, un Secrétariat d'État à la PME menait une très vigoureuse action sur le terrain. Aujourd'hui, le gouvernement considère que la déréglementation et le marché suffisent à satisfaire tous les besoins des PME » (Cahier, 1990).

Ainsi, à travers une approche théorique schématique dans laquelle s'inscrit le discours politique dominant, toute entreprise, sans discrimination de taille, semble confrontée, de manière identique aux autres, à un marché homogène.

* Le travail suivant a été réalisé afin de permettre l'émergence d'un débat autour des spécificités financières de la PME. À notre connaissance, ces dernières, que nous rencontrons régulièrement dans la vie économique quotidienne, n'ont fait l'objet, en Europe, que d'études parcellaires et n'ont jamais été intégrées dans un cadre théorique. Le premier objectif de cet article est de poser les bases d'une réflexion permettant de pallier cette insuffisance. Par ailleurs, la définition du concept de PME n'est pas abordée dans ce travail. Nous faisons nôtre pour l'instant la barrière de 500 salariés, telle qu'elle est justifiée par l'Institut d'études bancaires et financières (IEBE, 1989). 
Or, si de nombreux experts s'accordent pour penser qu'effectivement les grandes entreprises bénéficient de la dérèglementation et de la vague d'innovations financières (réduction du coût d'endettement, meilleure gestion des risques, soumission des organismes financiers à une plus forte concurrence pour l'ingénierie financière de très haut niveau), d'autres font remarquer que les PME subissent en général un effet d'éviction qui s'analyse surtout en terme de taille. En effet, il s'agit essentiellement de barrières quantitatives (seuils d'intervention) et qualitatives (sophistication des techniques) (Lagayette, 1987) se traduisant par des coûts d'accès aux marchés des produits innovants disproportionnés à l'échelle des PME.

De plus, une approche empirique suscite quelques questions. Les études périodiques du Crédit National ${ }^{1}$ montrent que les PME ont quasisystématiquement une rentabilité économique globale supérieure à celle des grandes entreprises, mais le coût moyen des capitaux qu'elles empruntent est plus élevé. Les études du Crédit National révèlent également que le levier d'endettement des PME (Dette financière totale/fonds propres) est plus élevé que celui des grandes entreprises. La structure de l'endettement est fortement discriminée par la taille: en 1988, l'endettement bancaire représente plus de $88 \%$ de leur endettement contre $77 \%$ pour les grandes entreprises. Il apparaît aussi une inégalité devant le financement qui présente un visage différent selon les secteurs: les taux d'intérêt affichés par les entreprises du tertiaire sont souvent inférieurs à ceux de l'industrie ${ }^{2}$. Mais la taille des entreprises n'est pas le seul facteur de disparité des conditions financières affichées par les PME. Ainsi, l'implantation géographique influe sur le coût du financement: dans les Ardennes, la Haute-Saône, la Creuse, les taux sont plus élevés que dans le Nord/Pas-de-Calais, et davantage encore qu'en île-de-France (Nau, 1989). La corrélation est forte entre la densité des établissements bancaires présents dans un département et le coût du financement. Cela dénote surtout que les entreprises moyennes subissant cette distorsion n'ont pas accès à un marché des capitaux où l'offre et la demande ne sont régulées que par les prix.

Il apparaît donc que la localisation géographique n'est pas neutre dans la structure et le coût du passif. Une réflexion empirique sur l'impact de la localisation et de l'utilisation de produits financiers innovants montre qu'il ne suffit pas qu'un produit existe dans un système financier national pour qu'il soit

1. Bulletin du Crédit National, $2^{e}$ trimestre $1987,3^{\mathrm{e}}$ trimestre 1989. Ces études portent sur la période 1983-1988 et définissent la rentabilité économique de manière comptable (Excédent Brut Économique / Actif Économique Brut), sans y intégrer le niveau de risque.

2. Étude INSEE sur la composition du passif des PME, 1988. 
utilisé de manière homogène à l'intérieur de celui-ci. Il en est ainsi pour les billets de trésorerie, titre de créances court-moyen terme sur des entreprises non bancaires, qui n'ont connu en Rhône-Alpes qu'un développement modeste. Seules cinq à six firmes y ont régulièrement recours localement, les autres étant de trop petite taille ou s'adressant directement à la Place de Paris. Ainsi, en décembre 1989, l'encours des billets de trésorerie en Rhône-Alpes s'élevait à 1,2 milliards de francs contre un encours national de 128 milliards (soit $1 \%$ ) (Belletante, Crozet et Gomez, 1990).

En confrontant cette réalité au tableau suivant (Froment et Karlin, 1988),

TABLEAU 1

Marché des encours de billets de trésorerie

\begin{tabular}{lcccccc}
\hline $\begin{array}{l}\text { Montant des encours } \\
\text { (avril 1988) }\end{array}$ & \multicolumn{2}{c}{$>00 \mathrm{MF}$} & $<500-100<$ & $<100 \mathrm{MF}$ \\
Siège à : & Paris & Hors & Paris & Hors & Paris & Hors \\
Nombre d'émetteurs & 28 & 5 & 38 & 12 & 23 & 10 \\
\hline
\end{tabular}

il apparaît que la concentration des volumes est plus forte que la concentration géographique. Serait-ce à dire qu'à partir d'un certain montant, les structures financières basées en Province ne sont plus capables de monter un dossier? Cela nous conduit à poser la question suivante : si nous considérons le marché des billets de trésorerie comme homogène à l'intérieur du marché financier français, comment expliquer un usage fortement différencié ? Quels sont les rôles et les fonctions des hommes non seulement à l'intérieur des entreprises (quelle est leur sensibilité à l'acceptation de l'innovation), mais aussi au sein des institutions offreuses de capitaux et productrices de services financiers? Quel est en outre l'impact de la structure de ces dernières ?

Alors que d'un côté le paradigme néoclassique réduit la firme à un agent économique homogène et rationnel, que la documentation financière ne s'intéresse qu'à la grande, voire la très grande entreprise, que les modèles d'analyse et d'évaluation financières n'intègrent pas d'outils spécifiques selon la taille de la firme ${ }^{3}$, que d'un autre côté des travaux mettent en évidence les spécificités

3. Le modèle d'analyse et de gestion financière pour les PME proposé par B. Kemkeng (RFG, avril-mai 1990, p. 73-77) ne nous semble pas relever d'une problématique spécifique, ne serait-ce que par sa référence tacite à un modèle de croissance équilibrée reposant sur une fonction de production à facteurs complémentaires et à générations d'équipements. 
de la petite entreprise (Julien et Marchesnay, 1988), nous nous proposons d'étudier les caractéristiques financières de la PME, à la fois de manière diachronique et synchronique. Pour cela, nous souhaitons définir une problématique du financement de la petite et moyenne firme en analysant son comportement au sein d'un espace financier, concept vecteur de l'offre globale en finance (offre de capitaux, de services, d'infrastructures). L'appropriation à un instant « $t$ » que la PME fera de cette offre définira pour cet instant « $t$ » son territoire financier. Que ce dernier intègre la dimension "temps" et la localisation géographique relève d'un emprunt classique à l'économie spatiale. Mais ces deux variables ne permettent pas à elles seules de définir l'ensemble des relations économiques permettant de définir tout d'abord l'espace comme contenu de plan (Perroux, 1969): il est nécessaire d'appréhender la stratégie des acteurs en fonction de leurs moyens et de leurs objectifs. Mais cette dernière va s'exprimer dans un espace identifié dans un deuxième temps à un champ de forces (Perroux, 1969); en effet, les relations entre les unités ne sont pas symétriques, il existe des influences émanant de groupes dominants. Comment s'exprime la dimension financière de la PME au sein de cet espace ? Peut-elle accéder (et comment) à la troisième catégorie d'espace distingué par F. Perroux, c'est-à-dire l'espace comme ensemble homogène se traduisant par un rapprochement des structures de prix et de production des unités, et permettant une régulation par les prix (exemple des actuels marchés de l'eurodollar, des euroobligations...)?

Les asymétries constatées ci-dessus, de manière empirique, entre PME et grandes entreprises, ne sont pas mises en évidence pour valider un traitement injuste des PME par les marchés financiers. D'une part, ce type de validation relève d'une démarche idéologique (d'ailleurs souvent présente dans les organisations patronales), d'autre part, ces asymétries peuvent refléter une réalité économique : un coût de financement plus élevé, par exemple, peut relever d'une appréciation d'un risque plus fort (Lloyd, Jahera et Goldstein, 1986). Le repérage des asymétries a pour fonction actuelle de nous aider dans la constitution d'un cadre référentiel du comportement financier de la PME.

Pour expliciter notre champ de recherche, nous commencerons par une présentation du modèle néoclassique et de son intégration dans un espace homogène, présentation assortie d'interrogations ayant pour objet d'aider à une approche plus précise de la problématique financière de la moyenne entreprise. Dans une deuxième partie, en nous appuyant sur des courants théoriques plus récents, nous préciserons notre approche de la Finance d'Entreprise à travers le concept de territoire financier, concept traduisant une appropriation et un vécu d'un espace financier hétérogène et polarisé. 


\section{Espace financier homogène et PME}

\subsection{Théorie financlère néoclassique et négation d'un territoire financier spécifique de la PME}

La théorie financière a été et est toujours dominée par une vision néoclassique présentant le processus de financement de l'entreprise comme la résultante d'un choix reposant sur un calcul rationnel. Cette rationalité est substantielle dans la mesure où le jugement de rationalité porte uniquement sur la décision. En effet, la rationalité de la décision est indépendante de la manière dont elle a été élaborée, le processus qui a abouti à cette décision étant le meilleur possible en termes de résultats obtenus (Favereau, 1989 ; Simon, 1976). Ainsi, la rationalité de l'agent économique débouche sur un comportement régi par un critère d'optimisation sous contraintes. Le marché sera alors la " prise en compte des décisions individuelles et les termes de l'échange sont ajustés jusqu'à ce que les décisions des individus soient mutuellement compatibles, globalement " (Arrow, 1976). Dans ce cadre, la finalité du processus de financement de la firme (véritable boîte noire) est réduite à son impact sur la maximisation du profit. L'espace financier de la firme est homogène et externe, celle-ci n'entretenant avec lui que des relations d'offre et de demande de capitaux. Les modèles de choix de financement sont dès lors déclinés comme relatifs à la structure présente du capital de la firme d'une part, et des espérances de flux futurs nets actualisés d'autre part : le territoire financier de la firme est réduit à des relations offre/demande régulées par un taux (Hamon et Malecot, 1986).

Cette approche nous conduit à deux interrogations. La première est liée au concept même de ressources financières. Les flux de capitaux résultant de leur offre et de leur demande ne sont pas homogènes : l'INSEE a montré qu'à l'intérieur d'un échantillon de petites et moyennes entreprises, la part des ressources propres dans le total des bilans est proportionnelle à la taille des entreprises. Elle représente ainsi $26 \%$ du total du bilan des petites entreprises, contre $41 \%$ pour les entreprises de plus de 500 millions de francs de chiffre d'affaires. Il ressort aussi de ces études que la répartition de l'endettement (court/long terme, endettement financier/endettement d'exploitation) est également influencée par la taille (Nau, 1989).

Une réflexion sur l'évolution de la structure du passif de l'entreprise (pourrait-on conceptualiser un cycle de vie de la structure financière ?) suppose une clarification du concept de capital, notamment dans la mesure où il faudra le quantifier. Si nous souhaitons en effet appréhender les composantes du territoire financier (au moins en tant que contenu de plan et de champ de forces), il est nécessaire non seulement d'identifier les offreurs et les demandeurs, mais 
aussi les flux. La conception «fonds de roulement » ou " capitaux permanents à long et moyen terme » est à exclure, premièrement parce que les contreparties ne correspondent à aucun élément réel ou monétaire, deuxièmement parce que l'exclusion des crédits bancaires à court terme ignore tout arbitrage entre long, moyen et court terme et qu'elle ignore la potentialité d'obtenir des financements stables dans la durée grâce à leur répétition (Raman, 1979). Le capital peut alors être approché comme l'addition des fonds propres, des dettes à long et moyen terme, des crédits bancaires à court terme et des dettes d'exploitation. Cette approche " pool de ressources " permet d'identifier les différentes sources de financement, mais n'autorise pas un rapprochement micro-macroéconomie.

Or ceci peut être préjudiciable si nous souhaitons confronter le territoire financier de l'entreprise avec l'espace lié à l'offre de capitaux. Dans quelle mesure le territoire financier d'une entreprise s'inscrit-il dans l'espace de l'offre ? De quelle manière peut-on établir, par exemple, une liaison entre la persistance d'une finance intermédiée au niveau de la région Rhône-Alpes (Belletante, Crozet et Gomez, 1990) et le territoire financier des entreprises Rhône-Alpines ? Le rapprochement micro-macroéconomie est méthodologiquement possible ; il en découle un concept de capital qui est l'addition des fonds propres nets, des dettes financées par l'épargne et des dettes financées par la monnaie (Raman, 1979). Satisfaisante par sa logique intégratrice, cette approche n'est pas sans soulever de nombreux problèmes au niveau microéconomique, ne serait-ce que celui de la détermination du coût des différentes ressources. Nous retiendrons simplement des lignes qui précèdent que toute analyse du comportement financier de l'entreprise moyenne ne peut faire l'économie d'une réflexion méthodologique sur le concept de capital.

Le deuxième type de questions suscitées par la définition du cadre financier néoclassique concerne les conséquences méthodologiques et analytiques de la rationalité substantielle. Nous avons rappelé que le territoire financier de l'entreprise y était réduit à des relations offre/demande de capitaux régulées par un taux. Ce dernier autorise une maximisation du rendement de l'investisseur en fonction d'un niveau de risque. La rentabilité issue des investissements doit rémunérer les apporteurs de fonds (compte tenu du risque encouru) et couvrir le coût du capital (Charreaux, 1988). Les bases de la théorie financière ${ }^{4}$ mettent donc en avant deux agents économiques : l'offreur (l'investisseur) de capitaux et le demandeur (généralement la firme).

4. Nous faisons plus particulièrement référence à Markowitz (1952), Modigliani et Miller (1958), Sharpe (1964), Lintner (1965), Black et Scholes (1973). 
Chaque catégorie d'agent est homogène dans ses comportements puisque agissant en fonction du couple rendement/risque analysé dans «des situations caractérisées par des aléas stationnaires, c'est-à-dire des aléas dont la loi de distribution est invariante, ou se modifie peu, au cours du temps " (Orléan, 1989). Le processus de décision s'appuie donc sur l'axiomatisation du critère de la maximisation de l'espérance subjective d'utilité. Le territoire financier qui en résulte est non seulement identique pour chaque firme, mais aussi composé d'agents qui ne peuvent être différenciés par des comportements économiques différents. Dans ces conditions, le marché des capitaux (et l'espace homogène qu'il induit) n'assure que des fonctions allocatives (Kaldor, 1987).

Or une vision empirique des mutations financières qu'ont vécues les économies occidentales depuis 1974 (Big Bang du New York Stock Exchange) nous montre que les marchés financiers ont des fonctions créatives (Kaldor, 1987). La révolution financière ayant essentiellement pour origine la volatilité des taux d'intérêt et des changes, l'internationalisation des économies et la vague de déréglementation des années 1970/1980 (Roure, 1990), a conduit à la création de nouveaux produits, de nouvelles structures de marchés et de nouveaux producteurs de services financiers.

Si d'un côté la vague d'innovations a eu au niveau macroéconomique un indéniable effet de globalisation (Around the world, around the clock) qui, par ses deux niveaux (intégration plus poussée des marchés existants et création de nouveaux marchés), a conduit à un meilleur arbitrage (aussi bien sur le plan national pour la France qu'international) entre les marchés du court terme et ceux du long terme (Gourlaquen, 1988), d'un autre côté cette vague d'innovations ne s'est pas diffusée uniformément. Il suffit de voir en Europe que seule la place de Londres a été présente quasiment sur tous les fronts de l'innovation, qu'il en est de même, mais avec une intensité moins forte, de Paris et de Luxembourg, que Amsterdam a poursuivi sa spécialisation sur les options pendant que les bourses allemandes se réveillaient et que l'ensemble du mouvement a quasi totalement ignoré les systèmes financiers espagnols, portugais, grecs et belges (Belletante et Crozet, 1990). Les fonctions créatives du marché ont donc effectivement mis en place, au niveau international, un véritable espace-ensemble homogène (Perroux, 1969) mais dont l'accès est sélectif, non seulement en raison de critères géographico-technico-réglementaires, mais aussi en raison de barrières économiques. Être émetteur sur le marché de l'Euro-CP demande la fourniture d'informations (autorisant l'efficience du marché) regroupées, par une notation communément acceptée, dans un rating élaboré par un des deux ou trois organismes auxquels les marchés font confiance ${ }^{5}$. Le coût

5. Il faut noter à ce propos qu'il s'agit d'un exemple d'autoréférence circulaire dont un des effets n'est pas de contribuer à l'équilibre des marchés, mais au contraire d'amplifier les déséquilibres ( $c f$. A. Orléan, 1989.) 
d'obtention de ce rating est tel qu'il écarte des marchés internationaux toutes les firmes qui ne sont pas de très grandes entreprises. L'exemple type est le retrait de Salomon, S.A. du marché de l'Euro-CP pour se réorienter vers le marché français des billets de trésorerie pour la simple et bonne raison que la société y obtenait un coût de financement moins élevé. Il apparaît donc que les gains de l'intégration ne sont pas les mêmes pour tous. De cette inégalité (qui se justifie partiellement en termes de coûts et de volumes) naît, à notre avis, une différenciation des territoires financiers des firmes, territoires qui ne sont pas intangibles comme nous l'avons vu avec l'exemple précédent.

Par ailleurs, certaines innovations financières ont eu pour mobile de répondre à des problèmes particuliers de taille des entreprises, modifiant ainsi l'espace financier global et induisant de facto de nouveaux territoires potentiels pour les firmes. Ainsi les créations de l'Unlisted Securities Market à Londres et du Second Marché à Paris ont eu pour objectif d'accueillir des petites et moyennes entreprises.

Mais cette offre d'une structure de marché n'est pas utilisée d'une manière homogène. Ainsi, le Second Marché français a une force d'attraction différente selon les places financières régionales :

Places

Bordeaux

Lille

Lyon

Marseille

Nancy

Nantes

Paris*
Nombre de sociétés introduites au 31/12/89

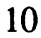

11

61

4

8

18

193

* Dont cinq sociétés étrangères.

Cette force d'attraction inégale s'explique en partie par l'offre d'ingénierie financière, car le classement est identique à celui que donnerait un bilan du nombre de banques, de conseillers financiers, de sociétés de bourse. Par contre, nous n'avons pas encore d'informations sur les demandes d'entreprises qui n'auraient pas été satisfaites.

Par ailleurs, malgré les efforts faits pour moderniser les marchés provinciaux, notamment celui de Lyon, la différence de fréquence d'opérations innovantes entre Paris et la province - au même titre que la différence d'utilisation de nouveaux produits financiers: par exemple, au 10 mars 1988, sur 37 émetteurs de MOF (« Multi Options Facilities »), seuls huit avaient leur siège social en province, et un seul était coté en province - est notoire, comme le montre le tableau 2. 
TABLEAU 2

Nombre d'opérations financières par an à Lyon par rapport à Paris

\begin{tabular}{|c|c|c|c|c|c|c|}
\hline & \multicolumn{2}{|c|}{1987} & \multicolumn{2}{|c|}{1988} & \multicolumn{2}{|c|}{1989} \\
\hline & $\begin{array}{l}\text { Cote } \\
\text { off. }\end{array}$ & $\begin{array}{c}2^{\mathbf{e}} \\
\text { marché }\end{array}$ & $\begin{array}{l}\text { Cote } \\
\text { off. }\end{array}$ & $\begin{array}{c}\mathbf{2}^{\mathbf{e}} \\
\text { marché }\end{array}$ & $\begin{array}{l}\text { Cote } \\
\text { off. }\end{array}$ & $\begin{array}{c}2^{\mathbf{e}} \\
\text { marché }\end{array}$ \\
\hline $\begin{array}{l}\text { Émissions en } \\
\text { numéraire }\end{array}$ & $3 / 105$ & $8 / 31$ & $4 / 81$ & $2 / 23$ & $2 / 77$ & $9 / 29$ \\
\hline ABSA & $0 / 2$ & $2 / 1$ & $0 / 4$ & $0 / 0$ & $1 / 7$ & $0 / 5$ \\
\hline OBSA & $2 / 29$ & $1 / 5$ & $0 / 4$ & $0 / 0$ & $0 / 7$ & $0 / 1$ \\
\hline Attribution & $0 / 33$ & $8 / 12$ & $3 / 17$ & $0 / 16$ & $1 / 30$ & $3 / 15$ \\
\hline Ob. Conver. & $0 / 5$ & $0 / 3$ & $1 / 18$ & $1 / 1$ & $1 / 14$ & $1 / 7$ \\
\hline Div. $\rightarrow$ titre & $0 / 33$ & $0 / 14$ & $0 / 45$ & $1 / 16$ & $2 / 55$ & $1 / 22$ \\
\hline
\end{tabular}

Note : Le tableau se lit de la façon suivante, en 1987, à la cote officielle, il y a eu à Lyon 3 émissions en numéraire contre 105 à Paris. Les ABSA sont des actions à bons de souscription d'action, les OBSA sont des obligations à bons de souscription d'action, la rubrique Obligation convertible comprend aussi les obligations échangeables et remboursables. La dernière ligne correspond au versement de dividendes avec des titres.

L'offre de structures de marché pour des entreprises de moyenne taille a pour objectif de les faire accéder à un circuit de financement spécialisé dont les fonctions sont l'allocation des capitaux, la valorisation des actifs financiers, la mutation des structures industrielles, auxquelles il faut rajouter celles traditionnellement affectées au marché secondaire (satisfaction des besoins de mobilité/liquidité des investisseurs) (Jacquillat et Solnik, 1989). Le tableau 3 montre que ces fonctions ne sont pas systématiques et qu'elles se traduisent même de manière anecdotique dans les chiffres. La volonté d'accéder au marché des capitaux (et le territoire financier en résultant) ne relève donc pas simplement d'une logique d'allocation des capitaux, mais de motivations plus diverses (Jaffeux et Le Guen, 1990).

TABLEAU 3

Répartition des motivations des sociétés lors d'une introduction en Bourse

\begin{tabular}{lccccc}
\hline $\begin{array}{l}\text { Intensité de } \\
\text { la motivation }\end{array}$ & Aucune & Peu élevée & Moyenne & Assez élevée & Élevée \\
\hline $\begin{array}{l}\text { Type de motivation } \\
\text { Succession }\end{array}$ & 40,8 & 9,2 & 13,3 & 9,2 & 14,3 \\
Notoriété & 2,0 & 5,1 & 13,3 & 31,6 & 42,9 \\
Financement & 7,1 & 8,2 & 15,3 & 23,5 & 38,8 \\
Patrimoine & 12,2 & 13,3 & 13,3 & 30,6 & 18,4 \\
Plus values & 22,4 & 26,5 & 15,3 & 18,4 & 7,1 \\
\hline
\end{tabular}


Par ailleurs, le choix entre Cote Officielle et Second Marché relève parfois d'une volonté de ne pas trop ouvrir (soit instantanément, soit temporairement) le capital de l'entreprise. Mais l'ensemble des critères ainsi évoqués ne relève pas du référentiel de la rationalité substantielle, ne serait-ce que parce qu'ils ne correspondent pas à une vision faisant de la firme une véritable «boîte noire » soumise à des contraintes d'environnement.

\subsection{Théorie de l'agence et territoire financier de la PME}

Devant l'incapacité des modèles néoclassiques à nous donner une clé de lecture des constatations empiriques réalisées, il faut nous tourner vers les approches qui ont levé l'hypothèse de la boîte noire.

Bien que ces approches maintiennent la firme soumise à une règle de maximisation du profit sous contrainte, la stratégie de financement de cette dernière intègre un nouvel élément : la compétition entre mandant et mandaté. Le choix financier (donc les relations de nature financière de l'entreprise) intègre un contenu informationnel. En effet, il est constaté qu'entre différents agents, il peut exister une asymétrie d'information, asymétrie qui conduit à des relations engendrant des coûts d'agence ${ }^{6}$. Pour toute recherche orientée vers les modalités d'usage de la structure d'offre de capitaux et de services financiers (dont la résultante à un instant « $t$ » représentera le territoire financier de l'entreprise), la conception des organisations liées à la théorie de l'agence nous apparaît comme essentielle. En effet, elle remet en cause l'identification et l'assimilation entreprise/actionnaires (Jensen et Meckling, 1976) : les actionnaires ne sont pas propriétaires de la firme mais des capitaux qu'ils ont apportés ${ }^{7}$. La séparation des fonctions de décision et d'assomption du risque est donc mise en évidence dans certaines organisations (Charreaux, 1988). Cette dernière nous conduit à une question : selon le degré de séparation de ces fonctions, n'y-a-t-il pas différenciation des relations établies entre la firme et les marchés de capitaux, et différenciation des influences subies? Autrement dit, selon le degré de séparation entre les fonctions de décision et d'assomption du risque, quelles sont les caractéristiques du territoire financier?

6. Nous faisons principalement référence ici aux travaux de Jensen et Meckling (1976), Fama (1980) et Klein (1983).

7. Cela conduit certains auteurs comme R. Shiller à distinguer plusieurs types de porteurs d'actions : ceux dont le comportement est conforme à la théorie néoclassique des marchés et ceux (noise traders) dont le comportement est influencé par les modes. 
Ces questions ouvrent également un champ d'investigation qui n'est pas sans interêt : comme, au cours de la vie de l'entreprise, ce degré de séparation est amené à évoluer, quelles sont les conséquences non seulement en termes de territoire, mais également en termes de structure de passif, de coût du capital, d'indépendance ? Répondre à ces questions permettrait d'aborder une typologie des stades financiers de l'entreprise, certains pouvant s'avérer plus efficaces que d'autres.

L'évolution de ce degré de séparation des fonctions est le reflet de l'existence de conflits entre dirigeants et actionnaires (Barnea, Hangen et Serbet, 1985). Ces derniers se manifestent plus particulièrement dans la politique de dividendes, support de la contradiction potentielle entre la maximisation de l'utilité du dirigeant et celle de la richesse des actionnaires. Il nous semble que de l'existence et de la résolution de tels conflits peuvent émerger des formes spécifiques du territoire financier d'une entreprise. Aussi, alors que $86 \%$ des entreprises de taille moyenne et non cotées ne distribuent pas de dividendes (Hirigoyen, 1984), la politique de distribution des dividendes est considérée comme un moyen de discipliner les dirigeants (Rozeff, 1982) : en effet, ce qui est distribué doit, surtout pour les entreprises en forte croissance, être recouvré sur le marché financier. "L'appel au marché financier constitue un moyen de contrôler la gestion de la firme. En vertu de cet argument, les sociétés à forte croissance qui font fréquemment appel au marché financier doivent normalement distribuer moins de dividendes, ce qui est conforme à l'observation » (Charreaux, 1988). L'asymétrie d'informations entre actionnaires et dirigeants engendre donc des modalités différentes de politiques financières, donc des relations et des forces différentes, qui d'ailleurs ne s'expriment pas uniformément.

Ainsi, nous considérons que le paiement du dividende en actions constitue un mode de résolution du conflit dirigeants/actionnaires, mais son utilisation en France est diverse selon les places, diversité qui est le reflet de comportements différents.

TABLEAU 4

Sociétés ayant réalisé un paiement de dividendes en titres

\begin{tabular}{lrrrr}
\hline & \multicolumn{2}{c}{ Cote officielle } & \multicolumn{2}{c}{ Second marché } \\
\cline { 2 - 5 } & \multicolumn{1}{c}{$\mathbf{1 9 8 8}$} & $\mathbf{1 9 8 9}$ & $\mathbf{1 9 8 8}$ & \multicolumn{1}{c}{$\mathbf{1 9 8 9}$} \\
\hline Paris & $45(10 \%)$ & $55(12 \%)$ & $13(7 \%)$ & $22(11,8 \%)$ \\
Lyon & $0(0 \%)$ & $2(3,4 \%)$ & $1(1,8 \%)$ & $5(3,2 \%)$ \\
Province & $3(2 \%)$ & $8(5,5 \%)$ & $5(4,7 \%)$ & $5(4,4 \%)$ \\
\hline
\end{tabular}

Note : Les pourcentages sont calculés par rapport au nombre total d'émetteurs d'actions. 
Par ailleurs, l'utilisation d'obligations convertibles ou d'obligations à bons de souscription est aussi un mode de résolution des conflits dirigeants/actionnaires, mais aussi de conflits pouvant opposer actionnaires et obligataires (Jensen et Smith, 1985 ; Jacquillat et Levasseur, 1984). La faible utilisation de ces produits dans la région Rhône-Alpes ( $c f$. tableau 2) peut s'expliquer alors autant par la structure du capital (présence fortement majoritaire des groupes familiaux dans le capital des entreprises cotées) que par une absence d'offre par les producteurs régionaux d'ingénierie financière.

Pour clore sur cet axe des relations actionnaires/dirigeants, nous voudrions souligner que son étude présentera des caractéristiques spécifiques à la PME. En effet, le développement du capital-risque et du capital-développement fait que l'identité actionnaire(s)-dirigeant(s) n'est plus une spécificité des grandes entreprises cotées. À côté du dirigeant-actionnaire-créateur apparaît, parfois dès la création, des actionnaires-accompagnateurs.

Leur présence a fait éclore, dans la structure des fonds propres des entreprises, divers produits et clauses juridiques qui apparaissent à nos yeux comme autant de moyens de discipliner les dirigeants-actionnaires. Il n'est en effet pas rare de voir certaines sociétés de capital-développement assortır leur participation de dividendes prioritaires avec maintien du droit de vote plus une clause d'un droit d'accès privilégié à l'information. Les petites et moyennes entreprises choisissant (avec quel degré de contraintes ?) un tel partenariat élargissent indéniablement leurs relations économiques financières, mais subissent une modification non négligeable de leurs rapports de force avec les offreurs de capitaux.

Nous venons donc de voir que la simple prise en compte de ce qui se passait dans la "boîte noire " offre des pistes d'analyse et de perception du territoire financier des entreprises, expression à un moment donné d'un usage de l'espace financier disponible. Mais la référence aux courants de la théorie de l'agence débouche généralement sur la grande entreprise et n'offre pratiquement pas de grille d'analyse selon la taille. À cette première remarque, il faut ajouter que la théorie de l'agence se maintient dans un cadre classique du marché qui suppose une répartition équilibrée de l'information ; par conséquent, elle réagit à la possible asymétrie d'information. Nous sommes toujours dans le cas où la rationalité de la firme est de nature substantielle. Tout se passe comme si la firme pouvait connaître, analyser et choisir en maximisant son intérêt, celui-ci étant confondu avec les flux actualisés de richesse gagnée ou économisée. Introduire la possibilité que les rationalités des acteurs (dirigeants, actionnaires, créanciers) ne convergent pas nécessairement ne remet pas en cause la cohérence a priori de la firme (Marksimovic, Sick et Zechner, 1989). Au stade actuel de notre analyse, pour aller plus loin et approcher au mieux les relations et les caractéristiques financières de la petite et moyenne entreprise, nous devons 
lever deux hypothèses: la rationalité illimitée substantielle des agents et la finalité du procès de financement, régi par la volonté de maximiser un intrant financier immédiat en minimisant les coûts présents et futurs.

\section{Vers une définition des composantes du territoire financier de la firme}

À partir du moment où nous levons l'hypothèse de la rationalité substantielle, nous ne pouvons qu'entrer dans la démarche proposée par H. A. Simon (1987 et 1989) au profit d'une conception procédurale de la rationalité. Une décision est issue d'une rationalité procédurale quand la procédure qui a conduit à ce qu'elle soit prise était la meilleure possible ${ }^{8}$, compte tenu des contraintes informationnelles. Or ce processus de décision s'exprime à l'intérieur d'une organisation entendue au sens large et caractérisée par un "ensemble de règles » et une « entité collective » (les règles formant un système pour des raisons juridiques et/ou des raisons de motivation) (Arrow, 1976). Or, l'abandon de l'hypothèse de la « boîte noire " (ce qui se passe à l'intérieur des entreprises n'est d'aucune importance pour comprendre ce qui se passe à l'extérieur) conduit à formuler une hypothèse inverse : ce qui se passe à l'intérieur est nécessaire pour comprendre ce qui se passe à l'extérieur (Favereau, 1989). Le cadre de cette réflexion théorique est reconnu sous le concept de «marché interne ». Nous notons d'ailleurs que la théorie de l'agence entre dans ce cadre tout en restant dans les limites de la rationalité substantielle.

Dans cette partie, nous souhaitons donc explorer le champ ouvert à la finance appliqué à la petite et moyenne entreprise en privilégiant le marché interne et la rationalité procédurale, de manière à montrer quelles pourraient être les composantes d'un territoire financier de l'entreprise.

\subsection{Marché interne, espace et territoire financiers}

La logique du marché interne permet d'intégrer le choix financier dans la culture de la firme, dans son équilibre fonctionnel interne, dans l'image et la perspective qu'elle se donne (Gomez, 1990). Dans quelle mesure les règles et les comportements à l'intérieur de la firme (et plus spécifiquement la moyenne entreprise) influent sur sa politique financière? Nous ne pouvons prétendre pour l'instant à une démarche exhaustive ; de ce fait nous nous contenterons de développer un certain nombre de pistes.

8. O. Favereau (1989) fait remarquer que le terme possible permet de " ne pas exclure la maximisation, même si un critère de satisfaction paraît infiniment plus plausible ». 


\subsubsection{Marchéisation et image interne}

La volonté politique et le discours théorique autour de la création de marchés boursiers adaptés aux moyennes organisations en Europe (Belletante et Crozet, 1990) se basent sur l'objectif théorique d'élargissement de l'espace financier offert aux entreprises à travers une diversification croissante des sources de financement et une mise en concurrence accrue des offreurs de capitaux. Nous avons déjà vu que les critères financiers n'étaient pas systématiquement mis en avant ( $c f$. tableau 3). Par ailleurs, la décision de s'introduire ne relève pas de motivations uniformes, ces dernières pouvant être modifiées selon le pourcentage de capital détenu par la famille avant l'introduction en Bourse (Jaffeux et Le Guen, 1990).

Tableau 5

Pourcentage de capital détenu par la famille avant l'introduction en Bourse

\begin{tabular}{lll}
\hline & \multicolumn{1}{c}{ Environ $50 \%$} & \multicolumn{1}{c}{$>\mathbf{7 0 \%}$} \\
\hline Motivations les plus élevées & - Notoriété & - Notoriété \\
& - Financement & - Liquidité du patrimoine \\
& - Plus-values & - Succession \\
Motivations les moins élevées & $\bullet$ Liquidité du patrimoine & $\bullet$ Financement \\
& - Succession & - Plus-values \\
\hline
\end{tabular}

L'introduction en Bourse, facteur de nouvelles relations financières et de modification des rapports de force entre les différents acteurs, relève donc de démarches fortement différenciées, celles-ci pouvant même aboutir à des retraits du marché financier dans un laps de temps relativement court (Binet Feutres, Cheval Blanc). La présence en Bourse pour une moyenne entreprise peut donc être aussi bien le résultat de la stratégie d'investisseurs souhaitant sortir, la recherche d'une reconnaissance d'un parcours d'entrepreneur, une face de la politique marketing ou de la politique sociale, qu'un choix délibéré d'accéder, dans les meilleures conditions possibles, à de nouvelles sources de financement. Que penser de sociétés moyennes présentes depuis plusieurs années sur le Second Marché et n'y ayant réalisé aucune opération ? Elles ont modifié leur territoire financier, mais ne l'ont pas utilisé. Quelles en sont les raisons ? Pourquoi cet arbitrage favorable au marché financier contre l'intermédiation n'a-t-il pas eu de suite ? Affirmer que les coûts de ce type de présence boursière n'engendrent aucun effet positif serait une erreur. En effet, la cotation sur une place financière peut être un moyen de renforcer la cohésion interne de l'entreprise. La mise en place de différentes sortes d'intéressement du personnel et l'affichage quotidien dans les locaux de l'entreprise du cours de bourse en sont 
des exemples. Le territoire financier peut donc résulter de la constitution et de la modification de l'image voulue de la firme (Marion, 1989) et la définition de ses composantes ne peut ignorer la référence au concept de réputation (Akerlof, 1985), une décision financière (accès à un marché, choix d'un produit innovant ...) procurant une renommée qui affectera ses choix futurs.

L'introduction du concept d'image dans la détermination des composantes du territoire financier de la firme ouvre une deuxième voie de réflexion. Dans la PME, le pool des fonds propres est moins réparti, moins atomisé entre différents actionnaires que dans la grande entreprise. De ce fait, la structure financière de la PME reflète correctement l'effort de l'entrepreneur, sa volonté de participation aux risques de la croissance. À ce titre, la structure financière constitue un signal en direction des différentes catégories de créanciers.

\subsubsection{Territoire financier et comportements des dirigeants}

Cette approche nous ouvre une voie de réflexion sur la diffusion des innovations financières dans les entreprises. Celles-ci apparaissent dans un système financier donné (national ou supranational), soit par l'action des autorités gourvernementales ou monétaires (innovations de politique), soit par le jeu des agents non étatiques, de nature financière ou non (innovations de système) (de Boissieu, 1986), ces derniers cherchant à répondre à des contraintes réglementaires pour maintenir ou améliorer leur position concurrentielle (Silber, 1977 ; Kane, 1988). Ces agents ont toujours été à notre connaissance de grandes organisations et ce n'est que dans un deuxième temps que les moyennes organisations y ont accès; la mise en œuvre de la nouveauté ne constitue plus alors qu'une innovation sur le plan de la structure utilisatrice. Pourquoi celle-ci est-elle donc prête à l'accueillir ? Cette question nous paraît d'autant plus importante que des travaux sur l'innovation financière ont montré que, généralement, la demande précède l'offre dans le processus d'innovation financière (Hardouin, 1973). En dehors des effets de réputation mentionnés ci-dessus, il nous semble intéressant de travailler sur les caractéristiques des dirigeants, notamment celles du directeur financier. Sur le marché financier lyonnais, de nombreux cas d'introduction en Bourse révèlent l'absence d'une direction financière autonome dans la structure préalable à l'accession au marché. La fonction apparât dans l'année qui suit et l'utilisation du marché financier (ainsi que la mise en place de nouveaux produits pour la firme) pourrait être en relation étroite avec les caractéristiques culturelles (âge, niveau et type de formation, origine professionnelle) du directeur financier. Nous noterons que ce dernier, par sa présence dans le monde de la finance, se construit sa propre image et maximise ainsi sa propre valeur sur le marché des responsables financiers. Notre étude du territoire financier des entreprises petites et moyennes se devra donc d'appréhender dans le marché interne de la finance les caractéristiques des dirigeants. 
Notre démarche rejoint en cela les travaux relatifs à la résolution de conflits entre des actionnaires et des dirigeants par l'intermédiaire de systèmes de rémunération et d'intéressement (Miller et Scholes, 1982).

Privilégier une logique de marché interne nous conduit à introduire dans la compréhension de la démarche financière de la moyenne entreprise le comportement de l'entrepreneur. Distinguons schématiquement l'entrepreneur à forte aversion pour le risque et celui favorable à des prises de risque élevé. Ce dernier va préférer les projets d'investissements les plus rémunérateurs qui sont aussi les plus risqués, et son comportement face au risque ne l'empêchera pas (au contraire) de financer ses projets par endettement. Et ce, contrairement à l'entrepreneur ayant une plus forte aversion au risque qui choisira des investissements plus sûrs qu'il préférera financer sur fonds propres. De ce fait, les entreprises à risque moyen ou faible tendent à sortir du marché des fonds prêtables, entrânant de fait un accroissement du degré de risque de ce dernier. Ce phénomène doit alors être confronté au principe théorique de sélection adverse (Stiglitz et Weiss, 1981). La structure oligopolistique du marché bancaire européen confère aux banques un caractère de groupe dominant (au sens de la théorie de l'agence). Or, les agents d'un groupe dominant sont à même de fausser un équilibre par les prix en mettant en œuvre des effets de rationnement (jeu sur les quantités). Ainsi, tout un chacun comprend que les banques ont intérêt à sélectionner les demandeurs sur le marché des fonds prêtables en fonction du risque de défaut qu'ils présentent. Cette sélection peut se faire par les variations des taux d'intérêt, mais ceci est une vision théorique : la domination des banques leur permet non seulement de fixer des prix plus élevés que ne l'exigerait l'équilibre du marché, mais aussi d'exclure les emprunteurs qu'elles jugent les moins fiables (Levratto, 1988).

Confronter le risque entrepreneurial (d'autant plus élevé que la firme est jeune) au principe de sélection adverse, est un élément nécessaire de la compréhension du territoire financier de la petite et moyenne entreprise. Les problèmes issus de cette confrontation apparaissent d'ailleurs comme un des éléments moteurs dans la création et le développement du capital-risque et des marchés boursiers de second rang (Geoffron, 1990). Cette constatation appelle deux commentaires. Premièrement, si le recours au financement direct par le marché financier est un moyen d'échapper aux contraintes du principe de sélection adverse, cela ne fait qu'accroître l'effet de domination pour les PME qui ne peuvent ou ne veulent pas y accéder. De plus, cet accès au marché financier n'est pas une garantie de l'éviction du principe de sélection adverse, dont la manifestation a été constatée sur les marchés boursiers (Stiglitz, 1985 et 1987). Deuxièmement, le marché des producteurs d'ingénierie en capital-risque ne nous semble pas refléter un degré de concurrence tel qu'il n'y existe pas un effet de sélection adverse. 
Au cours de cette section, nous avons mis en avant le comportement des dirigeants et soulevé l'existence ou non d'une fonction financière autonome dans l'entreprise. Il s'agit de deux variables permettant d'expliciter le territoire financier, deux variables déjà présentes dans la typologie des entreprises moyennes face aux conditions bancaires proposées par M. Dietsch (Dietsch, 1989). Cette dernière est notamment basée sur l'autonomie de la direction financière, l'indépendance des différentes fonctions financières (notamment la trésorerie) et l'analyse des relations avec les banques (rythme et objet des négociations, type de la relation - stabilité ou mise en concurrence).

Un des éléments de la décision et de la relation financières est, à notre avis, l'information. La gestion du territoire financier suppose la production d'informations, de la même manière que l'appropriation d'une partie de l'espace financier global nécessite l'acquisition d'informations. Dans le cadre de la théorie de l'agence et de la rationalité substantielle, nous avons déjà mentionné la pratique d'un droit privilégié à l'information exercé par certains entrants au capital d'entreprises moyennes. Il nous semble donc possible de penser qu'il doit y avoir une adéquation entre la distribution d'information et la stratégie de la firme.

À un territoire financier donné correspond-il un type de système informatif ? C'est ainsi que les entreprises de moyenne capitalisation cotées sur le Second Marché lyonnais ont un profil de communication typé : la communication financière est du ressort exclusif du dirigeant de l'entreprise (accessoirement du directeur financier), les budgets sont restreints (de 50 à $150 \mathrm{KF}$, et en moyenne inférieurs à $10 \%$ du budget communication commerciale) et alloués à une communication financière essentiellement destinée aux analystes et journalistes financiers, le petit actionnaire étant particulièrement oublié (Belletante, Boissier et Malleret, 1990).

\subsubsection{Territoire financier et actionnariat}

Cet oubli systématique et volontaire de l'information vis-à-vis du petit actionnaire nous conduit à nous interroger sur une typologie de l'actionnariat, typologie que nous avons déjà esquissée lors de notre rappel des apports de la théorie de l'agence. La distinction habituellement faite entre actionnaires internes (insiders) et actionnaires externes (outsiders) (Cobbaut, 1989) nous semble trop imprécise pour nous aider à préciser les composantes de l'espace financier des PME et signifier ainsi (entre autres possibilités) les signaux émanant de la politique de dividende. Plusieurs clés de lecture de l'actionnariat sont envisageables. 


\section{TABLEAU 6}

\section{Types d'actionnaires}

\begin{tabular}{ll}
\hline Actionnaires internes & Actionnaires externes \\
\hline - Fondateur/Dirigeant & - Fondateur/accompagnateur \\
- Dirigeant salarié : & - Accompagnateur $\rightarrow$ Plus-value en sortie \\
- cas normal & - Accompagnateur $\rightarrow$ Liquidité régulière \\
- cas RES* & - Accompagnateur $\rightarrow$ Pouvoir \\
- Salariés : & - Actionnaire « institutionnel » \\
- cas «normal » & - Actionnaire individuel \\
- cas RES** & \\
\hline
\end{tabular}

$1^{\text {re }}, 2^{e}, X^{\mathfrak{e}}$ génération de la famille du fondateur (ROMANET, 1984)

* RES = Reprise d'entreprise par les salariés

** Il est à noter que sur les dix sociétés cotées en Bourse en janvier 1990 et ayant mis en place un RES, six ont un chiffre d'affaires compris entre 210 et $488 \mathrm{MF}$ et trois parmi elles sont cotées sur une place provinciale (Le nouvel économiste, $\mathrm{n}^{\circ} 730$ du 26 janvier 1990).

Cette grille doit bien évidemment être croisée avec des critères propres aux étapes de la vie de l'entreprise (création, développement...), à sa stratégie financière (présence ou non sur le marché boursier) et à ses stratégies (impartition, croissance externe [Martinet, 1983], voire choix de l'hypofirme [Marchesnay, 1982] ...).

Vouloir connaître ce qui se passe à l'intérieur de la firme pour comprendre ce qui se passe à l'extérieur, et notamment comment s'est constitué son territoire financier, est une démarche qui ouvre de nombreuses pistes de recherche, caractéristiques d'une approche de la moyenne entreprise où les frontières fonctionnelles sont imprécises, mais également caractéristiques d'une problématique qui se refuse à réduire le choix financier au calcul économique. La poursuite de cette démarche consiste à repérer les éléments susceptibles de constituer puis d'expliquer les variations du territoire financier des entreprises moyennes dans les relations qu'elles entretiennent avec les composantes constitutives de l'espace financier global les environnant, voire même leur environnement politique 9 .

9. Nous précisons que cette démarche ne peut être qualifiée d'analyse du «marché externe ", ce concept relevant de l'attitude qui consiste à se désintéresser de ce qui se passe à l'intérieur des firmes pour comprendre ce qui se passe à l'extérieur. 


\subsection{Territoire financier et relations extérieures}

\subsection{1. Échange et territoires financiers}

Dans ses relations financières (relations ayant trait à la collecte des ressources et à la gestion des actifs financiers), la moyenne entreprise a à gérer une transaction d'achat. Bien que $\mathrm{O}$. Williamson ait essentiellement travaillé sur le rôle de la transaction dans le développement de la très grande entreprise (l'hyperfirme) (Williamson, 1975 et 1985), nous emprunterons à l'analyse de la transaction certains concepts en testant leur adéquation à une transaction financière d'acquisition.

Repérer les relations financières et les composantes du champ de force en résultant, peut s'articuler autour du concept de coût de transaction, concept largement divulgué dans la documentation économique. Le coût de la transaction financière sera la somme de plusieurs coûts. Hormis le coût explicite des capitaux collectés, il existe un coût de mise en œuvre du choix de financement, coût ayant de nombreuses composantes: analyse des solutions, prospection des offreurs de capitaux et des producteurs de services financiers, coût de surveillance de lá technologie financière, réalisation du contrat. Nous proposons également de définir un coût de mise en œuvre du choix de financement : coût du suivi administratif et juridique, coût de l'information propre au choix de financement. Il est évident que ce coût d'acquisition du capital n'est pas un coût propre aux PME. Mais il nous semble qu'il leur est plus spécifique, et ce essentiellement à cause de trois caractéristiques de l'échange financier entre la PME et son espace financier environnant. La première concerne l'importance des moyens spécifiques à mettre en œuvre pour réaliser l'échange financier. La reconduction d'un découvert n'implique pas la même mobilisation qu'une augmentation de capital réalisée lors d'une introduction en Bourse.

D'une manière générale, toute acquisition de capitaux implique pour la firme la fourniture d'informations précises sur sa contrainte de solvabilité et sur ses perspectives de rendement. Il est donc nécessaire de s'interroger sur la qualité du système d'information financière de la PME et de son adaptabilité aux attentes des différents fournisseurs de capitaux et aux différents produits de financement.

À ce sujet, les petites et moyennes entreprises sont sensibles à la sophistication des produits et à leur accessibilité, et ce, en termes d'«ancienrapide-bon marché-accès facile » contre "nouveau-lent-coûteux-accès difficile ", comme en termes de "sûr-souple " / " risqué-rigide " (Baumgartner et Jolibert, 1976). La deuxième caractéristique de l'échange financier de la PME est que l'asymétrie d'information (notamment en raison d'une asymétrie de technicité) est plus forte pour elle que pour la grande entreprise. La troisième 
caractéristique est la recherche, par la négociation, des conditions les plus favorables (Williamson 1985), afin d'avoir une meilleure maitrise de la transaction. Nous pensons pouvoir intégrer à cette troisième caractéristique le concept de dépendance, exprimant un rapport de force défavorable à la PME.

Les trois caractéristiques de l'échange financier de la PME sont à même de nous aider à définir son territoire financier. En fonction d'elles et du niveau des différentes composantes du coût de la transaction, certaines moyennes entreprises auront intérêt à développer des moyens spécifiques (direction financière, gestion de trésorerie...), développement qui n'est pas sans poser le problème du contrôle de la bureaucratie mise en place. D'autres choisiront plutôt de développer leurs relations financières à l'extérieur, ce qui leur posera le problème de la maîtrise de la transaction. Il apparaît donc que l'espace financier recouvre une problématique de pouvoir et de dépendance, cette dernière pouvant aboutir à un surcoût de financement ou à une inadéquation du montage proposé. Michel Marchesnay (1990) propose de mesurer le « degré de dépendance sur un flux d'échange à partir du taux de concentration, du degré de substituabilité et du caractère d'essentialité dans l'échange entre deux partenaires. "

En adaptant cette approche à la collecte de ressources financières, nous émettons l'hypothèse qu'il existe des correspondances entre le degré de dépendance financière et le territoire financier de la firme. Ainsi, une réflexion sur ce dernier ne peut éviter de prendre en compte la concentration des producteurs de services financiers et des offreurs de capitaux. Ne peut-on pas penser que la volonté de certaines entreprises moyennes de la Région Rhône-Alpes de se faire coter à Paris plutôt qu'à Lyon n'ait pas eu pour objectif d'éviter l'accroissement de la concentration de leurs relations financières sur la place de Lyon, ou de bénéficier du plus fort degré de concurrence (en tenant compte également d'une concurrence intraréseau) sur le marché parisien?

La substituabilité présente, quant à elle, plusieurs visages. Plus le projet et l'objet du financement sont standardisés, plus ils peuvent relever de l'intermédiation. Dans ce cas, l'entreprise se verra confrontée à une offre multiple. À l'inverse, plus l'objet du financement et son montage seront sophistiqués, plus il sera difficile de mettre en œuvre une substitution des produits et des offreurs. Les caractéristiques de l'échange financier de la PME sont telles que l'usage d'un montage financier sophistiqué, $s$ 'il se traduit dans un premier temps par une plus grande utilisation de l'espace financier, donc par un élargissement de son propre territoire financier, peut aboutir à une rigidification de ce dernier dans la mesure où la firme pourrait être dépendante de la technicité de l'organisme qui lui a vendu l'innovation financière.

N'y-a-t-il pas là une explication au plus faible développement de l'innovation financière dans la moyenne entreprise ? La caractéristique d'essentialité dans la mesure du degré de dépendance financière de la PME apparaît, quant à 
elle, dans une relation univoque. En effet, les mécanismes de prestige, illustrés par les informations habiles des tombstones publiées par la presse internationale, révèlent bien que les institutions financières cherchent à travailler en premier sur des opérations de prestige, sur lesquelles elles assoient leur renommée. La PME, à de très rares exceptions près, ne peut avoir accès (car n'en ayant forcément pas besoin) aux volumes exigés par de telles opérations et ne peut offrir aux organismes financiers autre chose que la réalisation de commissions. L'essentialité de la collecte de capitaux et l'asymétrie des rapports de force sont des caractéristiques de la dépendance financière des PME.

Face à cela, l'évolution du système financier français (ainsi que dans d'autres nations européennes) où la régulation de la concurrence (dont l'un des objectifs politiques avoués était de protéger les "petits») est abandonnée au profit d'une régulation par la concurrence (Rainelli, 1989), ne semble pas avoir modifié ni le degré d'essentialité, ni la dépendance. Faut-il attendre les effets de l'implantation de banques étrangères dans les régions françaises de manière à ce que la concurrence entre producteurs européens de services financiers s'accroisse d'une manière significative pour limiter le degré de dépendance des firmes moyennes et petites?

Cette prise en compte des caractéristiques de l'échange financier nous amène à nous interroger sur le rôle des intermédiaires financiers (entendu au sens le plus large). Il est à noter que la théorie financière s'exprimant dans le cadre du couple rationalité substantielle/marché externe admet la présence de l'intermédiation et justifie ses fonctions dans l'existence d'économies d'échelle, de possibilités de fractionnement et de groupage, d'atténuation des risques en raison de l'expertise et de la diversification, de possibilités de transformation de la nature juridique des actifs financiers (actif financier direct contre actif financier indirect) et de la transformation du terme (Cobbaut, 1987). L'ensemble de la démarche repose sur une diminution du coût relatif du produit et ne peut rendre compte que partiellement de la diversité des composantes de l'échange financier, telles que nous les avons évoquées précédemment. Mais il est possible d'aller un peu plus loin dans l'étude de l'échange financier de manière à mieux caractériser les composantes du territoire financier de la PME.

\subsubsection{Analyse réticulaire, espace et territoire financiers}

Jusqu'à présent, nous avons considéré les producteurs de services financiers et les offreurs de capitaux comme des entités indépendantes. Or, l'examen du marché montre qu'il existe entre eux non seulement des liens juridiques, mais aussi des liens culturels forts. L'impact de ces relations intra-offreurs de services financiers et de capitaux est un élément de l'espace financier environnant les PME, notamment sur le plan régional. En effet, elles sont à notre avis confrontées à trois réseaux. 
Le premier d'entre eux est un " réseau intégré ", constitué par les groupes bancaires nationaux et régionaux ${ }^{10}$. La volonté de ces derniers est d'offrir aux entreprises une gamme complète de produits financiers allant de la gestion des comptes courants à l'ingénierie de haut de bilan, en passant par la gestiondevises. Le degré de pression potentiel des membres de ce réseau intégré sur les PME est très fort et une logique d'arbitrage entre produits offerts à une même entreprise peut être mise en œuvre (par exemple, réduire la flexibilité du court terme pour s'orienter vers une restructuration du haut de bilan, opération génératrice d'une marge plus élevée).

Un second réseau se présente de manière atomisée et parcellaire. Il est constitué d'un ensemble de sociétés, petites ou moyennes, à vocation fortement régionale. Elles sont généralement spécialisées dans une activité d'ingénierie financière : conseil en création, conseil en développement, financement du démarrage, financement du développement, préparation et réalisation de l'accès au marché boursier. La spécificité élevée des produits proposés et la succession de ces derniers au cours du cycle de vie financier de la PME conduisent les producteurs desdits services à d'étroites relations entre eux et avec leurs clients. Celles-ci relèvent d'une approche clanique puisqu'elles ne correspondent ni à une logique de marché, ni à une logique de bureaucratie (Ouchi, 1980).

À l'intérieur de ce réseau clanique, il faut remarquer que les relations culturelles aboutissent à des liens juridiques : une étude rapide de la géographie des conseils d'administration montre de très nombreuses interférences entre les membres de ce réseau. En nous appuyant sur le cas de la région Rhône-Alpes, certains d'entre eux ont un rayon d'action national, voire international (SIPAREX), et d'autres sont des représentations régionales d'intervenants nationaux ou internationaux. Le troisième type de réseau, constitutif à la fois du territoire financier de la PME (demande) et de l'espace financier régional (offre), peut être qualifié de " techno-logique ". Il est en effet le résultat de la logique d'intervention technocratique de structuration économique des municipalités, départements, régions, chambres de commerce. Il se compose soit de structures de taille importante (instituts régionaux de participation, sociétés de développement régional) offrant une gamme élargie de services financiers, soit des structures très spécialisếes (dans le capital-risque notamment) ${ }^{11}$.

10. Les groupes dits régionaux étant par ailleurs l'objet de participations de la part de grands groupes nationaux : Lyonnaise de Banque - CIC, Banque de Vizille - GAN et Lyonnaise de Banque, Banque de Savoie - Crédit Lyonnais, pour ne citer que des exemples rhône-alpins.

11. Nous attirons l'attention sur le fait que le concept de réseau «techno-logique » est dû à $M$. Marchesnay; il a été identifié par celui-ci dans des zones d'activités où se développent, plus ou moins spontanément selon le volontarisme de l'aménageur public, de petites entreprises sans émergence de leader ou de contrôleur de l'ensemble de la filière. Nous affectons donc au concept de "techno-logique » un sens différent, plus axé sur la volonté des décideurs régionaux. 
Il est certain que les frontières de ces trois réseaux ne sont pas hermétiques et que leurs rapports ne sont pas simplement des rapports de concurrence. Nous constatons en effet un fort degré de coopération qui se caractérise notamment par la constitution de pools d'ingénierie et de financement, aboutissant, notamment sur les places financières régionales françaises, à des échanges de type clanique entre les trois réseaux. Ceci a une conséquence très précise dans le territoire financier des PME et dans la stratégie qui va en résulter.

Il y a, en effet, constitution au sein de la communauté financière d'un "common knowledge" (Dupuy, 1989) et nous pensons que la dimension autoréférentielle constatée sur les marchés boursiers (Orléan, 1988) est transférable au marché régional des capitaux. La PME est confrontée à un système fortement autoréférentiel puisqu'il " se définit par le fait que la grandeur par rapport à laquelle est évaluée la position des différents éléments le composant n'est pas une norme extérieure, comme dans les structures hétéroréférentielles, mais le produit même de l'interaction des stratégies élémentaires. Il s'ensuit que cette grandeur de référence est définie circulairement » (Orléan, 1989).

La conséquence directe, reflet régional du " peso problem 》 (Krasker, 1980), est que l'ensemble des intervenants se réfère à la même interprétation, à la même opinion moyenne, et le comportement optimal pour un expert est donc l'anticipation de cette opinion moyenne. À notre avis, l'existence de réseaux et le développement de relations claniques (qui ne sont donc pas simplement la base de l'un d'entre eux) inter-réseaux accroissent la dépendance (au sens analysé précédemment) des petites et moyennes entreprises.

La question qui se pose alors est de savoir si ce qui est présenté comme l'élargissement de l'espace financier (entre autres, l'intégration européenne) est à envisager comme une solution pour diminuer ce degré de dépendance, et comme un moyen d'accroissement de la concurrence. Et ce, d'autant plus qu'en même temps, la déréglementation ambiante conduit " la puissance publique, aujourd'hui nationale et demain européenne, à chercher à ne pas perturber avec ses interventions le jeu du marché au profit des petites entreprises » (Rainelli, 1989).

Nous sommes extrêmement prudents vis-à-vis de ce type d'anticipations, non seulement pour les rigidités entrevues précédemment dans l'espace et le territoire financiers, mais aussi parce que l'élargissement d'un espace économique n'est pas synonyme de plus grande concurrence.

Si nous regardons ce qui s'est passé sur le marché des transports aériens aux États-Unis, de nombreux économistes ont montré que la grande vogue de déréglementation ne s'est pas soldée par une homogénéisation de l'espace. Bien au contraire, on a assisté à une très forte structuration du trafic aérien : pour des 
raisons d'économies d'envergure, les compagnies aériennes ont eu intérêt à rabattre l'essentiel de leur trafic sur un aéroport donné et à développer, à partir de ce pôle, divers rayons de développement (théorie des moyeux et rayons hub and spoke-); une des conséquences directes est que dans la plupart des grands aéroports américains, une compagnie aérienne (donc un seul compétiteur dominant) réalise au moins $60 \%$ de l'activité (Bonnafous, 1990). Dans quelle mesure n'y a-t-il pas dans le domaine financier polarisation à grande échelle et radialisation sur le plan régional (Belletante, Crozet et Gomez, 1990) ?

La suppression des places boursières régionales (sur le plan de la cotation) constitue un effet de polarisation, mais une organisation locale devrait être mise en place et radialiser ainsi les espaces régionaux (à une polarisation technique correspondrait une radialisation d'ingénierie et de commercialisation).

Ce phénomène de radialisation, en s'appuyant sur les trois types de réseau existants, freinera la diffusion des effets positifs de la polarisation. Le risque est d'autant plus réel que les « moyeux » régionaux connaissent déjà une hiérarchie certaine, révélatrice d'asymétries, comme le révèlent les trois tableaux suivants.

\section{TABLEAU 7}

Opérateurs financiers ayant effectué des introductions au Second Marché à Lyon (1983-1989)

\begin{tabular}{lc}
\hline Opérateurs & Nombre d'introductions \\
\hline Lyonnaire de Banque et & 25 \\
Banque de Vizille & 20 \\
Crédit Lyonnais & 12 \\
Crédit Agricole/Segespar & 4 \\
Société générale & 3 \\
Banque Rhône-Alpes & 3 \\
Paribas & 27 \\
17 autres opérateurs & $101^{*}$ \\
\hline
\end{tabular}

* Ce nombre est supérieur au nombre d'introductions réalisées (67) car nous avons recensé les participations et plusieurs ont été conjointes (par exemple six pour Lyonnaise de Banque et Crédit Lyonnais. 
TABleaU 8

Gestion des services titres

\begin{tabular}{lccccc}
\hline & RM & $\begin{array}{c}\text { Comptant } \\
\text { marché }\end{array}$ & Second & Total & $\%$ \\
\hline Lyonnaise de Banque & 3 & 1 & 14 & 18 & 25 \\
Crédit Lyonnais & 3 & 3 & 12 & 18 & 25 \\
BNP & 0 & 4 & 6 & 10 & 14 \\
Siège social & 0 & 4 & 1 & 5 & 7 \\
12 organismes & 2 & 4 & 15 & 21 & 29 \\
\hline
\end{tabular}

TABLEAU 9

Pourcentage de participation des agents de change aux introductions du Second Marché à Lyon (1983-89)

\begin{tabular}{lr}
\hline Delore & 33 \\
Richard & 24 \\
Michaux & 21 \\
Girardet & 16 \\
3 autres charges & 6 \\
\hline
\end{tabular}

L'intégration de l'échange financier dans cette offre de services et de capitaux, structurée en réseau, montre de façon significative que la PME aura les plus grandes difficultés à maitriser sa transaction financière. Il se pose alors le problème de l'intégration de la Finance dans la stratégie de l'entreprise et dans celle de ses fournisseurs de capitaux. Ainsi, dans le secteur du transport de marchandises, la petite entreprise est devenue le pivot de la régulation "systémique » de la profession. "Sans elle, les prestations de distribution physique les plus sophistiquées sont rendues complètement inopérantes. Toute déréglementation doit donc prendre garde de ne pas priver les petites entreprises de ressources financières minimales. Des faillites en série obligeant à repenser entièrement la régulation "systémique " prévalante. Dilemme classique où les intérêts particuliers de court terme s'opposent parfois à l'intérêt général de long terme " (Paché, 1990). Aussi plus que jamais une déréglementation financière réalisée dans un système financier polarisé, radialisé et donc hétérogène, au lieu de servir l'intérêt idéologiquement qualifié de général, pourrait aboutir à de graves déséquilibres structurels au sein d'une économie. Considérer la Finance d'entreprise autrement que comme un processus d'allocation optimale des ressources financières, mais plutôt comme un espace d'expression de relations et de forces asymétriques nous apparaît comme un moyen d'anticiper de tels dérèglements. 


\subsubsection{Territoire financier et fiscalité}

L'approche des éléments constitutifs du territoire financier de la PME repose dans les paragraphes précédents sur l'identification d'agents économiques participant à la prise de décision de financement. Nous devons envisager l'existence d'agents totalement extérieurs au processus de financement et dont les actions dans l'environnement de l'entreprise peuvent influencer les choix de financement, et par là même l'usage de l'espace financier. Parmi ces agents « extérieurs », une attention toute particulière doit être accordée à l'État et aux collectivités locales, à travers la fiscalité.

De Alessi (1973) a montré que des arbitrages sont réalisés par les actionnaires majoritaires en fonction des écarts existant entre la fiscalité des revenus des personnes physiques et celle des sociétés. Les actionnaires majoritaires peuvent être conduits à effectuer des prélèvements soit directement (dividendes), soit indirectement (dépenses de "prestige » supportées par la firme). En tout état de cause, la capacité d'autofinancement nette de distribution est affectée et, a fortiori, la structure financière de la firme. Ceci conduit à penser que la structure du contrôle de l'entreprise, induisant un comportement fiscal donné, ne sera pas neutre sur le territoire financier de la firme (Gagnon et Suret, 1990).

L'impact de la fiscalité est illustré actuellement en France par deux exemples :

- La législation dite de l'intégration fiscale (dont l'objectif majeur est d'éviter, à l'intérieur d'un groupe, la juxtaposition de sociétés à déficit fiscal non employé et de sociétés lourdement imposables) permet de choisir librement les relations fiscales d'un groupe : la définition fiscale d'un résultat de groupe n'est pas neutre sur les structures de financement et les géographies du capital. Par exemple, des actionnaires majoritaires peuvent sortir du capital de leur société (en totalité ou partiellement), en cédant leurs participations à un groupe (industriel ou financier) qui, par l'intermédiaire d'un holding de contrôle détenu à au moins $95 \%$, financera l'acquisition en empruntant massivement. Les frais financiers en résultant seront couverts, redonnant au holding les dividendes versés par la filiale, et ce, dans les meilleures conditions fiscales. La fiscalité est ainsi un facteur motivant de restructuration, de transmission, et aboutit à un usage différent de l'espace financier.

- La législation fiscale française actuelle favorise les résultats réinvestis dans l'entreprise (imposés à $34 \%$ ) et pénalise les résultats distribués (imposés à $42 \%$ ). Le différentiel de taux de huit points s'applique sur l'exercice fiscal 1990. Dans quelle mesure accroîtra-t-il le nombre de PME non cotées à ne pas distribuer de dividendes? 
Les deux exemples précédents reflètent deux modalités fiscales différentes: l'intégration fiscale résulte du libre choix des entreprises, alors que la fiscalité des dividendes s'impose à toutes. Mesurer l'impact de la fiscalité sur le territoire financier de la firme devra intégrer des éventuels degrés de contraintes différentes.

\section{Conclusion}

Notre objectif de départ était d'examiner les principaux apports théoriques permettant d'identifier les spécificités financières de la PME. Le faire en s'appuyant sur le concept de territoire financier nous apparait heuristique. À travers les lignes précédentes, ce dernier apparaît comme un espace à quatre dimensions :

- un axe historique conjuguant le temps, la croissance des effectifs, du chiffre d'affaires et des moyens mis en œuvre ;

- un axe spatial repérant les divers fournisseurs de capitaux et de services financiers et leurs localisations géographiques ;

- un axe "produits financiers " matérialisant les différents supports de collecte de capitaux et les différents instruments de gestion financière, ce repérage ne pouvant pas être indépendant des caractéristiques du partenaire financier et des volumes mis en œuvre ;

- un axe "centre de décision financière " saisissant les mutations des structures, les caractéristiques et les comportements, non seulement des hommes pilotant la Finance d'entreprise, mais aussi des partenaires potentiels de la prise de décision financière (expert-comptable, conseiller financier, administrateur, actionnaire...).

Défini conceptuellement, le territoire financier doit maintenant être mesuré concrètement ${ }^{12}$ afin de déterminer les facteurs explicatifs de son évolution et ce, à l'intérieur de la mutation de l'offre de produits financiers et de capitaux, sur le plan régional, national et européen. Mais cette démarche présente d'autres implications. En effet, la connaissance du comportement financier des PME devrait permettre d'enrichir les outils de diagnostic et d'évaluation du risque financier. D'autre part, la compréhension du territoire financier ne peut se faire sans intégrer la dimension stratégique. Ce sera donc pour nous l'occasion de tester la spécificité des relations stratégie/finance à l'intérieur de la PME.

12. Une première série d'enquêtes est actuellement en cours. 


\section{Bibliographie}

Akerlof, G. (1985), An Economic Theorist's Book of Tales, Cambridge, Cambridge University Press.

Arrow, K. (1976), Les limites de l'organisation, Paris, Dunod.

Barnea, A., R. Hangen et L.W. Serbet (1985), Agency Problemis and Financial Contracting, Englewood Cliffs (N.J.), Prentice-Hall.

Baumgartner, G. et A. Jolibert (1976), "L'opinion des PME sur l'aide à l'exportation ", Revue française de gestion, mars-avril, $n^{\circ} 4$, p. 76-84.

Belletante B., V. Boissier. et S. Malleret (1990), «La communication financière d'entreprises moyennes cotées ", mémoire de PER, ESC Lyon.

Belletante, B. et Y. CROzet (1990), L'Europe monétaire et financière, ELLIPSES.

Belletante, B., Y. Crozet et P.Y. Gomez (1990), «Innovations et inerties dans l'espace financier régional », Congrès annuel de l'AIELF.

Borssieu, C. (de) (1986), «Quelques réflexions sur l'analyse économique des innovations financières ", Économie appliquée , n 3, p. 74-109.

Borssieu, C. (de) (1987), «La libéralisation financière et l'évolution du SME », dans Création d'un espace financier européen, Bruxelles, CEE, p. 67-95.

Bonnafous, A., (1990), "Mutations des systèmes de transport et radialisation de l'espace ", table ronde " Distance et analyse spatiale ", Chamonix, janvier.

CAHIER, M. (1990), Tribune de l'Expansion du 13 juin.

Charreaux, G.(1988), « La mise en cause du référentiel financier », IREA, Dijon, p. 67-86.

Charreaux, G. (1988), "La théorie positive de l'agence », IREA, Dijon, p. 115-168.

Cobbaut, R., (1987), Théorie financière, Paris, Economica.

Cobbaut, R.(1989), "Politique de dividende ", Encyclopédie de Gestion, Paris, Economica, p. 2105-2125

DE Alesi, L. (1973), «Private property and dispersion of ownership in large corporations ", The Journal of Finance, vol. $28, n^{\circ} 4$, p. 839-851, septembre.

Dietsch, M. (1989), "Les PME et les conditions bancaires", Revue d'économie financière, $\mathrm{n}^{\circ} 10$, p. 72-85.

DupuY, J.P. (1989), "Convention et common knowledge ", Revue économique, $\mathrm{n}^{\circ} 2$, p. $361-400$.

Favereau, O., (1989), "Marchés internes, marchés externes ", Revue économique, $\mathrm{n}^{\circ} 2$, mars, p. 273-328.

Froment, E., et M. KaRLIN (1988), Fonctions financières : comparaisons régionales et européennes, Lyon, 115 p.

GaGnon, J.M. et J.M. Suret (1990), “ Coûts d'agence, fiscalité et PME : aspects théoriques et tests empiriques«, Revue PMO, vol. 5, $\mathrm{n}^{\circ} 2$, octobre, p. 28-38.

Geofrron, P. (1990), "Le processus de formation d'une innovation financière : le capital-risque ", Thèse, Paris XIII.

GomeZ, P.Y. (1990), Cahier de recherche, ESC Lyon.

GoURLAQUEN, J.P. (1988), Les nouveaix instruments financiers, Paris, Vuibert.

Hamon, J. et J.F. Malecor (1986), "Contraintes financières et demande d'investissement ", Revue économique, septembre, vol. 37, $\mathrm{n}^{\circ}$ 5, p. 885-923. 
HARDOUIN, J.C. (1973 ), «L'apparition de l'innovation financière », thèse, Université de Rennes.

Hirigoyen, P. (1984), cité par G. Gallais-Hamonno et N. Mourgues dans « Organisation et décisions financières ", Revue d'économie financière, $\mathrm{n}^{\circ} 10,1989, \mathrm{p} .179-194$.

IEBE (1989), Caractéristiques et financement des PME en France, en Europe et dans les principaux pays industrialisés, Paris, Berger-Levrault.

Jacquillat, B. et M. Levasseur (1984), «Signaux, mandats et gestion financière », Finance, avril, vol. 5, $\mathrm{n}^{\circ} 1$, p. 5-83.

JaCQUillat, B. et B. Solnik (1989), Gestion de portefeuille et des risques, Paris, Dunod. JAFFEUX, C. et M. LE GuEN (1990), «La partition des sociétés sur des critères psychofinanciers est-elle rationnelle ? ", Analyse financière, $1^{\text {er }}$ trimestre , p. 64-81.

Jensen, M.C. et W.H. Mecklng (1976), "Theory of the firm : managerial behavior, agency costs and ownership structure ", Journal of Financial Economics, vol. 3, octobre, p. 305-360.

JENSEN, M.C. et L.W. SMITH (1985), « Stockholder, manager and creditor interests ».

Julien, P.A. et M. MARChesNAY (1988), La petite entreprise : principes d'économie et de gestion, Paris, Vuibert.

KALDOR, N. (1987), Économie et instabilité, Paris, Economica.

KANE, E.J. (1988), "Interaction of financial and regulatory innovation », AEA papers and proceedings, mai.

KRASKER, W.S. (1980), «The peso problem in testing the efficiency of forward exchange markets ", Journal of Monetary Economics, $n^{\circ}$ 6, avril, p. 269-276.

Lagayette, P. (1987), Perspectives de financentent de l'économie française, Paris, Commissariat général au Plan.

LeVratto N. (1988), « Sélection adverse, rationnement du crédit et fluctuations de l'activité économique », Paris, Latapses.

Lloyd, W.P., J.S. JAHERA et S.J. Goldstein (1986), «The relation between returns ownership structure and market value ", Journal of Financial Research, vol. 9, n², été , p. 171-177.

MARChESNAY, M. (1982), "Pour un modèle d'hypofirme », Entreprise et Organisation, Paris.

MARCHESNAY, M. (1988), «Quelle techno-logique ? », Revue internationale PME, vol. 1, $\mathrm{n}^{\circ} 2$.

MARCHESNAY, M. (1990), « La transaction, outil d'analyse stratégique », dans Mélanges en l'honneur du Professeur Lassègue.

Marion, G. (1989), Les images de l' entreprise, Paris, Editions d'Organisation.

MARKsimovic, V., G. SicK et J. ZeCHNER (1989), « Comment on forward markets, stocks markets and the theory of the firm », Journal of Finance, juin, vol. 44, n ${ }^{\circ}$, p. 428-512.

Martinet, A.C. (1983), Stratégie, Paris, Vuibert.

MILLER, M.H. et M.S. SCHOLES (1982), « Executive compensations, taxes and incentives », dans Financial Economics : essays in honor of Paul Cootner, Prentice-Hall, p. 179-201.

NAU, V. (1989), « Des structures financières disparates », Option Finance, $\mathrm{n}^{\circ}$ 52, février, p. $18-19$. 
ORLÉAN, A. (1988), «L'autoréférence dans la théorie keynésienne de la spéculation », Cahiers du CREA, 11 et "Comportements mimétiques et diversités des opinions sur les marchés financiers ", Actes du Colloque "Théorie économique et perturbations des marchés financiers ", Paris, p. 119-144.

Orléan, A. (1989), " Pour une approche cognitive des conventions économiques », Revue économique, $\mathrm{n}^{\circ} 2$, p. 241-272.

Ouchl, W. (1980), "Markets, bureaucraties and clans", Administrative Science Quarterly, vol.25, mars.

PACHÉ, G. (1990), «Stratégies d'adaptation de la petite entreprise », RFG, avril-mai, p. $52-61$.

Perroux, F. (1969), L'économie du XXe siècle, Paris, PUF.

PETEERs, T. (1989), « Intégration financière, pourquoi ? », dans Espace financier européen et coopération monétaire, Bruxelles, Economica, p. 11-16.

Rainelli, M. (1989), «L'analyse économique et les PME », dans Les PME dans les systèmes économiques contemporains, Bruxelles, De Boeck Université.

Raman, J.P. (1979), " Pour une définition élargie du capital : du paradoxe à l'utopie ", Cahiers de l'IAE, Lille, p. 2-23.

ROMANET, Y. (1984), «Les différentes générations d'actionnaires », document ESC Lyon.

ROURE, F. (1990), «L'innovation financière : fondements, opportunités et risques ", CREFIB , p. 1-14.

Roure, F. et R. Tabatoni (1988), La dynamique financière, Paris, Economica.

ROZEFF, M.S. (1982), "Growth, Beta and Agency costs as determinants of dividend pay out ratios ", Journal of Financial Research, automne, vol. 5 , p. 249-259.

SILBER,W. (1977), «Financial Innovation : a linear programming approach », Journal of Banking and Finance, $\mathrm{n}^{\circ} 1$.

Simon, H.A. (1987), "From substantive to procedural rationality », dans Latsis Method and Appraisal in Economics, Cambridge University Press, p. 129-148.

Simon, H.A. (1989), « Rational decision making in business organization », American Econonic Review , septembre.

STIGLITZ, J.E. (1985), " Credit markets and control of capital ", Journal of Money Credit and Banking, vol. 17, $\mathrm{n}^{\circ}$ 2, mai, p. 133-152.

Stiglitz, J.E. et A. WeIss (1981), "Credit rationing in markets with imperfect information ", American Economic Review, juin, $n^{\circ} 71$, p. 313-409.

Williamson, O.E. (1975), Markets and Hierarchies : Analysis and Antitrust Implications, New York, MacMillan, Free Press.

Williamson, O.E.(1985), The Economic Institutions of Capitalism : Firms, Markets, Relational Contracting, New York, MacMillan, Free Press. 